\title{
Ambientes e temporada de vocalização da anurofauna no Município de Espigão do Oeste, Rondônia, Sudoeste da Amazônia - Brasil (Amphibia: Anura)
}

\author{
Paulo Sérgio Bernarde
}

Biota Neotropica $v 7$ (n2) - http://www.biotaneotropica.org.br/v7n2/pt/abstract?article + bn01507022007

\author{
Recebido em 08/08/06 \\ Versão reformulada recebida em 15/02/07 \\ Publicado em 01/05/07
}

Centro de Ciências Biológicas e da Natureza, Campus Floresta, Universidade Federal do Acre - UFAC, 69980-000 Cruzeiro do Sul, AC, Brasil

e-mail: paulobernarde@hotmail.com,www.ufac.br

\begin{abstract}
Bernarde, P.S. Calling period and environments of anuran fauna in Municipality of Espigão do Oeste, Rondônia, Southeastern of Amazonian - Brazil (Amphibia: Anura). Biota Neotrop. May/Aug 2007 vol. 7, no. 2 http://www.biotaneotropica.org.br/v7n2/pt/abstract?article+bn01507022007 ISSN 1676-0603.

A survey of the anuran fauna was carried out in the Jaburi farm $\left(11^{\circ} 35^{\prime}-11^{\circ} 38^{\prime} \mathrm{S}\right.$ and $\left.60^{\circ} 41-60^{\circ} 45^{\prime} \mathrm{W}\right)$, Municipality of Espigão do Oeste, Rondônia State, Brazil, analysing spatial distribution and calling activity period of the species in primary forest and pasture areas. Field work at Jaburi farm was carried out from February 2001 until March 2002. For sampling of the species, different environments were investigated extensively throughout searching frogs in breeding sites and catching by pitfalls traps. A total of 47 species out of ten families were recorded: Aromobatidae (1), Brachycephalidae (2), Bufonidae (4), Centrolenidae (1), Cycloramphidae (1), Dendrobatidae (1), Hylidae (23), Leiuperidae (1), Leptodactylidae (8) and Microhylidae (5). New registers for Rondônia State were the following species: Dendropsophus acreanus, Trachycephalus resinifictrix, Chiasmocleis bassleri e C. ventrimaculata. The majority of species called during rainy period (October to March), and there was a significant correlation between calling activity and rainfall. More anuran species were found within the forest than on pasture areas, showing that deforestment may decrease number of species.

Keywords: amphibians, anurans, habitat use, breeding, calling activity.

Resumo

Bernarde, P.S. Ambientes e temporada de vocalização da anurofauna no Município de Espigão do Oeste, Rondônia, Sudoeste da Amazônia - Brasil (Amphibia: Anura). Biota Neotrop. May/Aug 2007 vol. 7, no. 2 http://www.biotaneotropica.org.br/v7n2/pt/abstract?article+bn01507022007 ISSN 1676-0603.

Um levantamento da anurofauna foi realizado na Fazenda Jaburi $\left(11^{\circ} 35^{\prime}-11^{\circ} 38^{\prime} \mathrm{S}\right.$ e $\left.60^{\circ} 41-60^{\circ} 45^{\prime} \mathrm{W}\right)$, Município de Espigão do Oeste, Estado de Rondônia, Brasil, analisando a distribuição espacial e período da atividade de vocalização das espécies registradas em áreas de floresta primária e de pastagem. O trabalho de campo na Fazenda Jaburi foi realizado entre fevereiro de 2001 e março de 2002. Para amostragem das espécies, diferentes ambientes foram investigados intensivamente por procura ativa e também por armadilhas de interceptação e queda. Um total de 47 espécies, pertencentes a dez famílias foi registrado: Aromobatidae (1), Brachycephalidae (2), Bufonidae (4), Centrolenidae (1), Cycloramphidae (1), Dendrobatidae (1), Hylidae (23), Leiuperidae (1), Leptodactylidae (8) e Microhylidae (5). Novos registros para o Estado de Rondônia são as seguintes espécies: Dendropsophus acreanus, Trachycephalus resinifictrix, Chiasmocleis bassleri e C. ventrimaculata. A maioria das espécies apresentou atividade de vocalização durante o período chuvoso (outubro a março), e houve correlação significativa entre a atividade de vocalização e a pluviosidade. Foi encontrada a maior riqueza de espécies nas áreas de floresta do que nas de pastagem, podendo ser um indicativo de que o desmatamento pode provocar a diminuição da riqueza de espécies.
\end{abstract}

Palavras-chave: anfíbios, anuros, uso de hábitat, reprodução, atividade de vocalização. 


\section{Introdução}

A anurofauna da Amazônia ainda é insuficientemente conhecida, sendo poucas regiões amostradas (Azevedo-Ramos \& Galatti 2002, Silvano \& Segalla 2005). Nestas, uma grande riqueza de espécies de anfíbios anuros é registrada (Duellman 1978, De La Riva et al. 2000, Azevedo-Ramos \& Galatti 2002); em algumas regiões como o Parque Nacional da Serra do Divisor (Acre) foram registradas 124 espécies (Souza et al. 2003). No estado de Rondônia existem poucas publicações sobre inventários ou biologia de anfíbios anuros (e.g., Heyer 1977, Vanzolini 1986, Vitt \& Caldwell 1994, Bernarde et al. 1999, Brandão 2002). Devido à expansão agropecuária e extração de minérios e madeira, a paisagem natural florestal vêm sendo modificada rapidamente e diversas espécies podem estar desaparecendo antes mesmo de serem descritas (Haddad \& Prado 2005). Anfíbios anuros são animais sensíveis a alterações ambientais, como destruição dos hábitats, alterações climáticas e poluição, sendo um grupo de importância como bioindicadores da integridade ambiental (e.g., Vitt et al. 1990; Heyer et al. 1994).

Em Espigão do Oeste (RO), Bernarde et al. (1999) apresentaram uma lista preliminar com 31 espécies de anfíbios em uma localidade (Fazenda Jaburi). Nesse estudo foi coletada uma nova espécie de Proceratophrys (Giaretta et al. 2000), sendo o primeiro registro do gênero para a Amazônia. A colonização antrópica neste município começou cerca de 35 anos atrás (Oliveira 2002), sendo comum e constante o desmatamento especialmente para a pecuária e a exploração de madeira (Vanzolini 1986, Oliveira 2002). Recentemente, iniciou-se a exploração ilegal de diamantes em reserva indígena. A carência de informações sobre a diversidade de anfíbios anuros e a velocidade da modificação da paisagem e impactos ambientais que vêm ocorrendo na região denota a urgência na realização de inventários da fauna e da flora do Estado.

O presente trabalho tem como objetivo apresentar uma lista atualizada das espécies de anfíbios anuros da Fazenda Jaburi em Espigão do Oeste (RO), e informações sobre os ambientes de reprodução e a temporada de vocalização das espécies.

\section{Material e Métodos}

O estudo foi realizado entre fevereiro de 2001 e março de 2002 , na Fazenda Jaburi $\left(11^{\circ} 35^{\prime}-11^{\circ} 38^{\prime} \mathrm{S}\right.$ e $\left.60^{\circ} 41-60^{\circ} 45^{\prime} \mathrm{W}\right)$, Município de Espigão do Oeste (RO) (Figura 1). A região encontra-se no

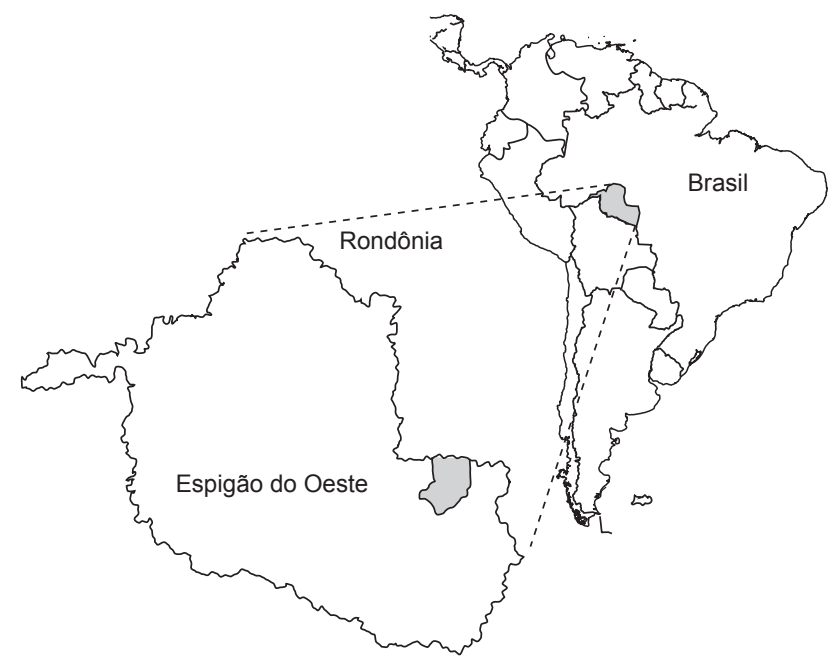

Figura 1. Mapa da América do Sul com a localização de Espigão do Oeste (RO) - Brasil.

Figure 1. Map of South American with localization of Espigão do Oeste (RO) - Brazil.
Domínio Equatorial Amazônico “Amazônia” (sensu Ab’Saber 1977), com vegetação do tipo Floresta Ombrófila Aberta Submontana (Oliveira 2002). A altitude da região varia em torno de 280 metros. A pluviosidade média anual é de $2100 \mathrm{~mm}$, com dois períodos distintos durante o ano: chuvoso (de outubro a março correspondendo a $88 \%$ dos índices pluviométricos) e seco (de abril a setembro). A temperatura média anual é de $26^{\circ} \mathrm{C}$. Os dados climáticos durante o período desse estudo foram obtidos na Prefeitura Municipal de Espigão do Oeste.

A Fazenda Jaburi situa-se no km 32 da Rodovia do Calcário, perfazendo uma área de 4000 hectares, dos quais 50\% correspondem à reserva legal (mata de terra firme). Os ambientes visitados foram: floresta (rio, riachos temporários, ambientes distantes de corpos d'água, poças temporárias e poças permanentes); borda de floresta (riachos, açudes, brejos e poças temporárias); pastagem (poças temporárias, açudes, ambientes distantes de corpos d'água). A área amostrada totaliza cerca de 1000 hectares.

O trabalho de campo iniciou-se em fevereiro de 2001 (reconhecimento da área e dos ambientes) e a amostragem regular foi realizada entre abril de 2001 e março de 2002. As espécies foram registradas através de dois métodos (ver Heyer et al. 1994) em áreas de floresta e de pastagem: procura ativa em ambientes propícios para a reprodução de anuros e armadilhas de interceptação e queda ("pitfall"). Quinzenalmente os ambientes de reprodução foram visitados entre as 18:00 e 23:00 horas, onde todas as espécies em atividade de vocalização foram registradas. As armadilhas de interceptação e queda constam de tambores de plásticos (200 L), enterrados a cada dez metros e ligados por uma cerca de lona de um metro de altura. Um conjunto de armadilhas consta de quatro tambores interligados por lona. Estes foram instalados em três tipos de ambientes: interior de mata, distante (500 m) de corpos d'água permanente; $100 \mathrm{~m}$ de um rio (cerca de $4 \mathrm{~m}$ de largura e 0,80 $\mathrm{m}$ de profundidade), dentro de mata; em área de pastagem, distante de corpos d'água permanentes. Foram seguidas as recomendações apresentadas por Cechin \& Martins (2000).

Foi utilizado o teste de Spearman (Zar 1984) para verificar possível correlação entre a riqueza de espécies de anuros em atividade de vocalização e pluviosidade ao longo do ano.

A identificação dos espécimes foi feita com base em descrições das espécies na literatura, (Duellman 1978, Rodríguez \& Duellman 1994, De La Riva et al. 2000). Os espécimes testemunhos foram depositados nas seguintes coleções herpetológicas: Coleção "CFBH" da Universidade Estadual Paulista - UNESP, em Rio Claro (SP), Coleção "AAG-UFU" da Universidade Federal de Uberlândia (MG) e da Coleção "UFAC" da Universidade Federal do Acre, em Rio Branco (AC).

\section{Resultados}

Foram registradas 47 espécies de anuros pertencentes às seguintes famílias: Brachycephalidae (2 espécies), Hylidae (23), Centrolenidae (1), Leptodactylidae (8), Leiuperidae (1), Cycloramphidae (1), Dendrobatidae (1), Aromobatidae (1), Bufonidae (4) e Microhylidae (5) (Tabela 1). A maioria das espécies (38) foi encontrada em floresta, seguido de borda de floresta (35) e pastagem (20) (Tabela 1).

Das 47 espécies registradas, 39 foram observadas em atividade reprodutiva (vocalização, presença de desova ou de girinos) nos ambientes estudados (Tabela 1). A maioria das espécies utilizou poças temporárias (16), seguido de poças permanentes (10), açudes (9), riachos (5), ambiente terrestre (5), rio (4), brejo (2), riacho temporário (1), ocos de árvores (1) e formigueiros de saúva (Atta sp.) (1).

O período de vocalização de 33 espécies foi monitorado ao longo do estudo (Tabela 2; Figura 2). A maioria das espécies foi registrada vocalizando nos meses de outubro a março (Tabela 2; 
Tabela 1. Distribuição das espécies de anfíbios anuros em floresta (FLO), borda de floresta (BFL) e área de pastagem (AAB) e nos ambientes de reprodução em Espigão do Oeste (RO) - Brasil.

Table 1. Anurans species distribution in forest (FLO), forest border (BFL) and pasture areas (AAB) and in breeding environments in Epigão do Oeste (RO) Brazil.

\begin{tabular}{|c|c|c|c|c|}
\hline Espécie & FLO & BFL & AAB & Ambiente \\
\hline \multicolumn{5}{|l|}{ AROMOBATIDAE } \\
\hline Allobates gr. marchesianus * & $\mathrm{X}$ & $\mathrm{X}$ & & $\mathrm{Te}, \mathrm{Pt}, \mathrm{Ri}$ \\
\hline \multicolumn{5}{|l|}{ BRACHYCEPHALIDAE } \\
\hline Eleutherodactylus fenestratus (Steindachner 1864)* & $\mathrm{X}$ & $X$ & $X$ & $\mathrm{Te}$ \\
\hline Eleutherodactylus gr. conspicillatus $*$ & $\mathrm{X}$ & $\mathrm{X}$ & & $\mathrm{Te}$ \\
\hline \multicolumn{5}{|l|}{ BUFONIDAE } \\
\hline Chaunus marinus (Linnaeus 1758)* & $\mathrm{X}$ & $\mathrm{X}$ & & Ro \\
\hline Chaunus schneideri (Werner 1894)* & & & $\mathrm{X}$ & $\mathrm{Aç}$ \\
\hline Rhaebo guttatus (Schneider 1799) & $\mathrm{X}$ & $\mathrm{X}$ & & \\
\hline Rhinella margaritifera (Laurenti 1768)* & $\mathrm{X}$ & $\mathrm{X}$ & $\mathrm{X}$ & $\mathrm{Pt}$ \\
\hline \multicolumn{5}{|l|}{ CENTROLENIDAE } \\
\hline Gen. sp.* & $\mathrm{X}$ & $\mathrm{X}$ & & Ro \\
\hline \multicolumn{5}{|l|}{ CYCLORAMPHIDAE } \\
\hline $\begin{array}{l}\text { Proceratophrys concavitympanum } \\
\text { Giaretta, Bernarde \& Kokubum, 2000* }\end{array}$ & $\mathrm{X}$ & & & Pp, Ri, Rt \\
\hline \multicolumn{5}{|l|}{ DENDROBATIDAE } \\
\hline Adelphobates quinquevittatus (Steindachner 1864) & $\mathrm{X}$ & & & \\
\hline \multicolumn{5}{|l|}{ HYLIDAE } \\
\hline Dendropsophus acreanus (Bokermann 1964)* & $\mathrm{X}$ & $\mathrm{X}$ & $X$ & $\mathrm{Pt}$ \\
\hline Dendropsophus bokermanni (Goin 1960)* & $\mathrm{X}$ & & & $\operatorname{Pr}$ \\
\hline Dendropsophus leucophyllatus (Beireis 1783) * & & $\mathrm{X}$ & $\mathrm{X}$ & $\mathrm{Pp}, \mathrm{Pt}, \mathrm{Aç}$ \\
\hline Dendropsophus microcephalus (Cope 1886)* & & $\mathrm{X}$ & $\mathrm{X}$ & $\mathrm{Pt}, \mathrm{Aç}$ \\
\hline Dendropsophus minutus (Peters 1872)* & $X$ & $\mathrm{X}$ & $\mathrm{X}$ & $\mathrm{Pt}, \mathrm{Aç}$ \\
\hline Dendropsophus schubarti (Bokermann 1963)* & $\mathrm{X}$ & $\mathrm{X}$ & & $\mathrm{Pp}, \mathrm{Pt}$ \\
\hline Hypsiboas boans (Linnaeus 1758)* & $\mathrm{X}$ & $\mathrm{X}$ & & Ro \\
\hline Hypsiboas cinerascens (Spix 1824)* & $\mathrm{X}$ & $\mathrm{X}$ & & $\mathrm{Pr}, \mathrm{Br}$ \\
\hline Hypsiboas fasciatus (Günther 1858)* & $\mathrm{X}$ & $\mathrm{X}$ & & $\operatorname{Pr}$ \\
\hline Hypsiboas geographicus (Spix 1824)* & $\mathrm{X}$ & $\mathrm{X}$ & $\mathrm{X}$ & Ro, Ri, Pp, Aç \\
\hline Hypsiboas lanciformis (Cope 1871)* & $\mathrm{X}$ & $\mathrm{X}$ & & $\mathrm{Pp}, \mathrm{Ri}$ \\
\hline Hypsiboas multifasciatus (Günther 1859)* & & $\mathrm{X}$ & $\mathrm{X}$ & $\mathrm{Br}$ \\
\hline Osteocephalus taurinus Steindachner $1862 *$ & $\mathrm{X}$ & $\mathrm{X}$ & & $\operatorname{Pr}$ \\
\hline Osteocephalus sp. & $\mathrm{X}$ & & & \\
\hline Phyllomedusa camba De la Riva 2000 “1999”* & $\mathrm{X}$ & $\mathrm{X}$ & $\mathrm{X}$ & $\mathrm{Pp}, \mathrm{Pt}, \mathrm{Aç}$ \\
\hline Phyllomedusa tomopterna (Cope 1868)* & $\mathrm{X}$ & $\mathrm{X}$ & & $\mathrm{Pp}, \mathrm{Pt}$ \\
\hline Phyllomedusa vaillanti Boulenger 1882 * & $\mathrm{X}$ & $\mathrm{X}$ & & $\mathrm{Pp}, \mathrm{Aç}$ \\
\hline Scinax garbei (Miranda-Ribeiro 1926)* & $\mathrm{X}$ & $\mathrm{X}$ & & $\mathrm{Ri}$ \\
\hline Scinax nebulosus (Spix 1824)* & & $\mathrm{X}$ & $\mathrm{X}$ & $\mathrm{Aç}$ \\
\hline Scinax ruber (Laurenti 1768)* & $\mathrm{X}$ & $\mathrm{X}$ & $\mathrm{X}$ & $\mathrm{Pp}, \mathrm{Pt}, \mathrm{Aç}$ \\
\hline Scinax sp. $*$ & & & $\mathrm{X}$ & $\mathrm{Pt}$ \\
\hline Trachycephalus resinifictrix (Goeldi 1907)* & $\mathrm{X}$ & & & Oc \\
\hline Trachycephalus venulosus (Laurenti 1768)* & & & $\mathrm{X}$ & $\mathrm{Pt}$ \\
\hline \multicolumn{5}{|l|}{ LEIUPERIDAE } \\
\hline Engystomops petersi Jiménez-de-la-Espada* & $\mathrm{X}$ & $\mathrm{X}$ & $\mathrm{X}$ & $\mathrm{Pp}, \mathrm{Aç}$ \\
\hline \multicolumn{5}{|l|}{ LEPTODACTYLIDAE } \\
\hline Leptodactylus andreae (Müller 1923)* & $\mathrm{X}$ & $\mathrm{X}$ & $\mathrm{X}$ & $\mathrm{Te}$ \\
\hline Leptodactylus fuscus (Schneider 1799)* & & $\mathrm{X}$ & $\mathrm{X}$ & $\mathrm{Pt}$ \\
\hline Leptodactylus lineatus (Schneider 1799)* & $\mathrm{X}$ & $\mathrm{X}$ & $\mathrm{X}$ & Fo \\
\hline Leptodactylys mystaceus (Spix 1824) & $\mathrm{X}$ & $\mathrm{X}$ & & \\
\hline Leptodactylus pentadactylus (Laurenti 1768)* & $\mathrm{X}$ & $\mathrm{X}$ & $\mathrm{X}$ & $\mathrm{Pt}$ \\
\hline
\end{tabular}


Tabela 1. Continuação...

\begin{tabular}{|c|c|c|c|c|}
\hline Espécie & FLO & BFL & $\mathbf{A A B}$ & Ambiente \\
\hline Leptodactylus rhodomystax Boulenger, 1884 & $\mathrm{X}$ & & & \\
\hline Leptodactylus wagneri (Peters 1862)* & $\mathrm{X}$ & $\mathrm{X}$ & & $\operatorname{Pr}$ \\
\hline Leptodactylus (Lithodytes) sp. * & $\mathrm{X}$ & $\mathrm{X}$ & & $\mathrm{Te}$ \\
\hline \multicolumn{5}{|l|}{ MICROHYLIDAE } \\
\hline Elachistocleis ovalis (Schneider 1799)* & & & $\mathrm{X}$ & $\mathrm{Pt}$ \\
\hline Chiasmocleis bassleri Dunn, 1949 & $\mathrm{X}$ & & & \\
\hline Chiasmocleis ventrimaculata (Andersson 1945) & $\mathrm{X}$ & $\mathrm{X}$ & & \\
\hline Ctenophryne geayi Mocquard, 1904 & $\mathrm{X}$ & $\mathrm{X}$ & & \\
\hline Hamptophryne boliviana (Parker 1927)* & $\mathrm{X}^{*}$ & & & $\mathrm{Pt}$ \\
\hline Total de espécies & 38 & 35 & 20 & \\
\hline
\end{tabular}

* = Espécie observada em atividade reprodutiva nos ambientes (Indivíduos vocalizando ou presença de desova e girinos): Ro = Rio; Ri = Riacho; Rt = Riacho temporário; $\mathrm{Pt}=$ Poça temporária $; \mathrm{Aç}=\mathrm{Açude} ; \mathrm{Te}=$ Terrestre $; \mathrm{Pr}=$ Poça temporária próxima a rio; $\mathrm{Br}=\mathrm{Brejo} ; \mathrm{Pp}=\mathrm{Poça} \mathrm{permanente} ; \mathrm{Oc}=\mathrm{Ocos}$ de árvores com água armazenada; e Fo = Dentro de formigueiros.

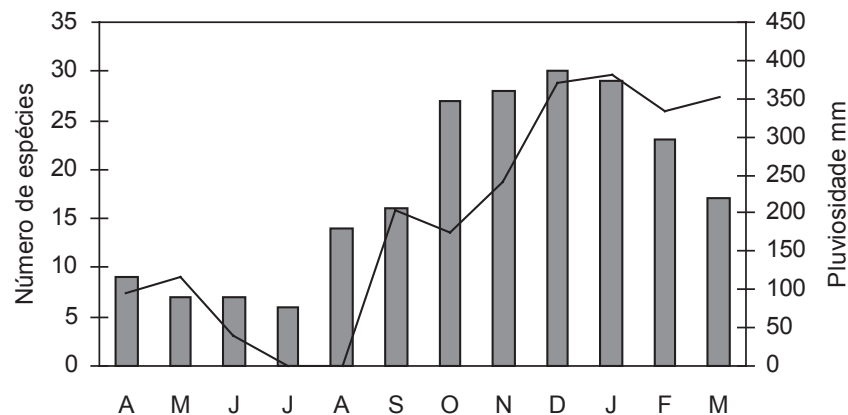

Figura 2. Relação entre riqueza de anuros em atividade de vocalização (barras) e a pluviosidade (linha) durante entre abril de 2001 e março de 2002 em Espigão do Oeste (RO) - Brasil.

Figure 2. Relation between richness anurans in calling activity (bars) and the rainfall (line) during the period of april 2001 from march 2002 in Espigão do Oeste (RO) - Brazil.

Figura 2), coincidindo com o período em que ocorreram os maiores índices pluviométricos. Algumas espécies como Chaunus marinus e Hypsiboas boans foram encontradas nos meses mais secos. Houve correlação significativa entre o número de espécies em atividade de vocalização e a pluviosidade $(\mathrm{r}=0,8211 ; \mathrm{P}=0,0011 ; \mathrm{N}=12)$. Agregações de Osteocephalus taurinus e Trachycephalus venulosus foram observadas em reprodução explosiva (sensu Wells 1977) durante duas noites consecutivas (em meses diferentes) após chuva torrencial.

\section{Discussão}

A riqueza de espécies é semelhante ao registrado em outras localidades amazônicas (ver Azevedo-Ramos \& Galatti 2002), que podem chegar até 124 espécies (Souza et al. 2003). Em comparações de riqueza de anuros na Amazônia deve-se levar em conta, além do esforço amostral, também a área de estudo, pois quanto maior a área, um maior número de ambientes ela abrangerá e conseqüentemente poderá apresentar um maior número de espécies (ver Azevedo-Ramos \& Galatti 2002). O esforço amostral foi concentrado em uma área de 1.000 hectares, ocorrendo outras espécies (e.g., Phyllomedusa hypochondrialis e Physalaemus cuvieri) em áreas próximas (cerca de $20 \mathrm{~km}$ ) de ecótono com o Cerrado. Foram registradas pela primeira vez para o Estado de Rondônia (segundo Frost 2004) as seguintes espécies: Dendropsophus acreanus, Trachycephalus resinifictrix, Chiasmocleis bassleri e C. ventrimaculata. Diferente da Mata Atlântica (ver Haddad \& Prado 2005), a anurofauna da Amazônia brasileira apresenta poucos endemismos, com cerca de 13 espécies consideradas endêmicas (Azevedo-Ramos \& Galatti 2002). Proceratophrys concavitympanum pode ser considerada uma espécie endêmica do Sudoeste da Amazônia brasileira, pois até o momento só foi registrada nessa região (Bernarde et al. 1999, Giaretta et al. 2000).

Algumas espécies (e.g., Dendropsophus leucophyllatus, Hypsiboas geographicus, Phyllomedusa camba e Scinax ruber) apresentaram grande plasticidade ambiental, ocorrendo em diferentes ambientes tanto no interior da floresta como nas pastagens, inclusive nos açudes artificiais destinados ao gado. Outras espécies (e.g., Trachycephalus resinifictrix e Leptodactylus lineatus) apresentam modos reprodutivos mais especializados, dependendo de locais específicos. Trachycephalus resinifictrix é encontrada principalmente em dossel, e se reproduz exclusivamente em ocos de árvores preenchidos com água (Hödl 1990). Leptodactylus lineatus apresentou atividade de vocalização dentro de formigueiros de saúva (Atta sp.); esta associação já observada anteriormente (ver Schlüter \& Regös 1981, Lamar \& Wild 1995).

A maioria das espécies foi registrada em áreas de floresta ou borda, havendo um decréscimo nas áreas de pastagens. Algumas espécies florestais aparentemente são prejudicadas com a conversão de florestas em pastagens (e.g., Adelphobates quinquevittatus, Proceratophrys concavitympanum, Phyllomedusa tomopterna e Trachycephalus resinifictrix), enquanto outras espécies de áreas abertas são favorecidas (e.g., Leptodactylus fuscus, Scinax ruber e Trachycephalus venulosus) (ver Aichinger 1991, Tocher 1998, Brandão 2002, Haddad \& Prado 2005, Caldwell \& Araújo 2005). Embora algumas espécies consigam colonizar ambientes alterados na Amazônia, outras não encontram condições favoráveis, principalmente devido à perda dos hábitats de reprodução (e.g., Aichinger 1991, Tocher 1998). Além disso, algumas espécies de anuros de serapilheira especialistas quanto ao alimento, podem ter sua ocorrência limitada em ambientes alterados devido à perda de suas presas (Aichinger 1991).

A atividade de vocalização da maioria das espécies de anuros ocorreu durante os meses chuvosos, característica de comunidades em regiões onde a chuva é restrita a seis ou menos meses durante o ano (Aichinger 1987). Exceções foram Hypsiboas boans e Chaunus 
Tabela 2. Espécies de anfíbios anuros em atividade de vocalização durante o período de abril de 2001 a março de 2002 em Espigão do Oeste (RO) - Brasil.

Table 2. Anurans species in calling activity during the period of april 2001 from march 2002 in Espigão do Oeste (RO) - Brazil.

\begin{tabular}{|c|c|c|c|c|c|c|c|c|c|c|c|c|}
\hline Espécies $\ /$ Meses $\Rightarrow$ & $\mathbf{A}$ & $\mathbf{M}$ & $\mathbf{J}$ & $\mathbf{J}$ & $\mathbf{A}$ & $\mathbf{S}$ & $\mathbf{O}$ & $\mathbf{N}$ & $\mathbf{D}$ & $\mathbf{J}$ & $\mathbf{F}$ & $\mathbf{M}$ \\
\hline A. gr. marchesianus & & & & & & & + & + & + & + & + & \\
\hline E.fenestratus & & & & & + & + & + & + & + & + & + & \\
\hline E. gr. conspicillatus & + & + & + & + & + & + & + & + & + & + & + & + \\
\hline C. marinus & + & + & + & + & + & + & & & & & & \\
\hline R. guttatus & & & & & & & & + & + & & & \\
\hline R. margaritifer & & & & + & + & & & & & & & \\
\hline Gen. sp. & & & & & & & + & + & + & + & + & + \\
\hline P. concavitympanum & & & & & & & + & + & + & + & & \\
\hline D. acreanus & & & & & & + & + & + & + & + & + & + \\
\hline D. leucophyllatus & & & & & & + & + & + & + & + & + & + \\
\hline D. microcephalus & + & & & & + & + & + & + & + & + & + & + \\
\hline D. minutus & & & & & & & & & + & + & + & + \\
\hline D. schubarti & & & & & & & + & + & + & + & + & + \\
\hline H. boans & & & + & + & + & + & + & & & & & \\
\hline H. geographicus & + & + & + & + & + & + & + & + & + & + & + & \\
\hline H. lanciformis & + & + & & & + & + & + & + & + & + & + & + \\
\hline H. multifasciatus & + & + & + & & + & + & + & + & + & + & + & + \\
\hline O. taurinus & & & & & & & + & & & & & \\
\hline$P$. tarsius & + & & & & & & + & + & + & + & + & + \\
\hline P. tomopterna & & & & & & & + & + & + & + & & \\
\hline P. vaillanti & & & & & + & + & + & + & + & + & + & \\
\hline S. garbei & & & & & & & & & + & + & + & + \\
\hline S. nebulosus & & & & & + & + & + & + & + & + & + & \\
\hline S. ruber & + & + & + & & & + & + & + & + & + & + & + \\
\hline T. resinifictrix & + & + & + & + & + & + & + & + & + & + & + & \\
\hline T. venulosus & & & & & & & & + & & & & \\
\hline E. petersi & & & & & & & + & + & + & + & & \\
\hline L. andreae & & & & & + & + & + & + & + & + & + & + \\
\hline L. fuscus & & & & & + & + & + & + & + & + & + & + \\
\hline L. lineatus & & & & & & & & + & + & + & & \\
\hline L. pentadactylus & & & & & & & + & + & + & + & & \\
\hline E. ovalis & & & & & & & + & + & + & + & + & + \\
\hline H. boliviana & & & & & & & & & + & + & + & \\
\hline Total de Espécies & 09 & 07 & 07 & 06 & 14 & 16 & 25 & 26 & 28 & 27 & 22 & 15 \\
\hline
\end{tabular}

marinus, que ocorrem principalmente nos meses mais secos, de modo semelhante ao registrado em outros estudos (Duellman 1978, Aichinger 1987). A pluviosidade e a subseqüente disponibilidade de corpos d'água e microhábitats terrestres com alta umidade são provavelmente os mais importantes fatores ambientais influenciando a reprodução dos anuros na Amazônia (Aichinger 1987, Hödl 1990).

\section{Agradecimentos}

A Lílian Cristina Macedo Bernarde pelo auxílio nas atividades de campo e de laboratório. Aos amigos Marcelo Kokubum e Reginaldo A. Machado pelo auxílio em algumas saídas de campo. A Célio Haddad e Moisés Barbosa de Souza pela confirmação de algumas identificações. Aos proprietários da Fazenda Jaburi Eduardo Garcia, Celso Garcia e família pelo apoio logístico. A Carlos Alberto Bernarde e família pelo apoio logístico em Espigão do Oeste. Ao Centro de Conservação e Manejo de Répteis e Anfíbios - RAN do IBAMA pelas licenças de coleta concedida (Processo 02001.006649/00-60; Licenças 246/2000DIFAS/DIREC e 021/02-RAN). A Yeda S. de Lucena Bataus (RAN) pela orientação sobre a solicitação e relatórios referentes à licença de coleta. A CAPES pela bolsa de estudo concedida.

\section{Referências Bibliográficas}

AB' SABER, A.N. 1977. Os domínios morfoclimáticos da América do Sul. Boletim do Instituto de Geografia da Universidade de São Paulo 52:1-21.

AICHINGER, M. 1987. Annual activity patterns of anurans in a seasonal neotropical environment. Oecologia 71:583-592.

AICHINGER, M. 1991. Faunal deficit of anurans in tropical farmland of Amazonian Peru. Alytes 9:23-32.

AZEVEDO-RAMOS, C. \& GALATTI, U. 2002. Patterns of amphibian diversity in Brazilian Amazônia: conservation implications. Biological Conservation 103:103-111. 
BERNARDE, P.S., KOKUBUM, M.C.N., MACHADO, R.A. \& ANJOS, L. dos. 1999. Uso de hábitats naturais e antrópicos pelos anuros em uma localidade no Estado de Rondônia, Brasil (Amphibia: Anura). Acta Amazonica 29:555-562.

BRANDÃO, R.A. 2002. Avaliação ecológica rápida da herpetofauna nas reservas extrativistas de Pedras Negras e Curralinho, Costa Marques, RO. Brasil Florestal 74:61-73.

CALDWELL, J.P. \& ARAÚJO, M.C. 2005. Amphibian faunas of two eastern amazonian rainforest sites in Pará, Brazil. Occasional Papers Sam Noble Oklahoma Museum of Natural History 16:1-41.

CECHIN, S.Z. \& MARTINS, M. 2000. Eficiência de armadilhas de queda (pitfall traps) em amostragem de anfíbios e répteis no Brasil. Revta. Bras. Zool. 17:729-740.

DE LA RIVA, I, KOHLER, J, LOTTERS, S. \& REICHLE, S. 2000. Ten years of research on Bolivian amphibians: Update checklist, distribution, taxonomic problems, literature and iconography. Revista Española de Herpetologia 14:19-164.

DUELLMAN, W.E. 1978. The biology of an equatorial herpetofauna in Amazonian Ecuador. University of Kansas, Museum of Natural History Miscellaneous Publications 65:1-352.

FROST, D.R. (ed.). 2004. Amphibians species of the world 3.0 - an online reference. American Museu of Natural History. Disponível em <http:// research.amnh.org/herpetology/amphibia/index.php> Acesso em 20/01/ 2007.

GIARETTA, A.A., BERNARDE, P.S. \& KOKUBUM, M.C.N. 2000. A new species of Proceratophrys (Anura: Leptodactylidae) from the Amazon Rain Forest. J. Herpetol. 34:173-178.

HADDAD, C.F.B. \& PRADO, C.P.A. 2005. Reproductive modes in frogs and their unexpected diversity in the Atlantic Forest of Brazil. BioScience 55:207-217.

HEYER, W.R. 1977. Taxonomic notes on frogs from the Madeira and Purus rivers, Brasil. Pap. Avulsos Zool. 31:141-162.

HEYER, W.R., DONNELLY, M.A., MCDIARMID, R.W., HAYEK, L.C. \& FOSTER, M.S. 1994. Measuring and monitoring biological diversity: Standard methods for amphibians. Smithsonian Institution Press, Washington.

HÖDL, W. 1990. Reproductive diversity in Amazonian lowland frogs. Fortschr. Zool. 38:41-60.
LAMAR, W.W. \& WILD, E.R. 1995. Comments on the natural history of Lithodytes lineatus (Anura: Leptodactylidae), with description of the tadpole. Herpetol. Nat. Hist. 3:135-142.

OLIVEIRA, O.A. 2002. Geografia de Rondônia - espaço e produção. Dinâmica Ed. e Dist. Ltda, Porto Velho.

RODRIGUEZ, L.O. \& DUELLMAN, W.E. 1994. Guide to the Frogs of the Iquitos Region, Amazonian Peru. Asociación de Ecologia y Conservación, Amazon Center for Environmental Education and Research, and Natural History Museum, The University of Kansas, Lawrence.

SCHLÜTER, A. \& REGOS, J. 1981. Lithodytes lineatus (Schneider, 1799) (Amphibia: Leptodactylidae) as a dweller in nests of the leaf cutting ant Atta cephalotes (Linnaeus, 1758) (Hymenoptera: Attini). AmphibiaReptilia 2:117-121.

SILVANO, D.L. \& SEGALLA, M.V. 2005. Conservation of brazilian amphibians. Conserv. Biol. 19:653-658.

SOUZA, M.B., SILVEIRA, M., LOPES, M.R.M., VIEIRA, L.J.S., GUILHERME, E., CALOURO, A.M. \& MORATO, E.F. 2003. Biodiversidade no Estado do Acre: Conhecimento atual, conservação e perspectiva. Revista T\&C Amazônia 1:45-56

TOCHER, M. 1998. Diferenças na composição de espécies de sapos entre três tipos de floresta e campo de pastagem na Amazônia central. In Floresta Amazônica: Dinâmica, Regeneração e Manejo (C. Gascon \& P. Moutinho, eds.). Ministério da Teconologia e Ciência, Instituto de Pesquisas da Amazônia, Manaus, p.219-232.

VANZOLINI, P.E. 1986. Levantamento herpetológico da área do Estado de Rondônia sob a influência da rodovia Br-364. Polonoreste/Ecologia Animal. DF. Relatório de Pesquisa ${ }^{\circ} 1, \mathrm{CNPq}$, Brasília.

VITT, J.P., WILBUR, H.M. \& SMITH, D.C. 1990. Amphibians as harbingers of decay. BioScience 40:418.

VITT, L.J. \& CALDWELL, J.P. 1994. Resource utilization and guild structure of small vertebrates in the Amazon forest leaf litter. J. Zool. 234:463-476.

WELLS, K.D. 1977. The social behavior of anuran amphibians. Animal Behavior 25:666-693.

ZAR, J.H. 1984. Biostatistical analysis. Prentice-Hall International, Inc. 2. Ed., New Jersey. 